\title{
Solid-State Reaction and Vacancy-Type Defects in Bilayer Fe/Hf Studied by the Slow Positron Beam
}

\author{
K. Yamada ${ }^{1}$, T. Sasaki1, T. Nagata1 ${ }^{1}$, I. Kanazawa ${ }^{1 *}$, R. Suzuki², T. Ohdaira ${ }^{2}$, K. Nozawa ${ }^{3}$, \\ F. Komori ${ }^{3}$ \\ ${ }^{1}$ Department of Physics, Tokyo Gakugei University, Tokyo, Japan \\ ${ }^{2}$ The National Institute of Advanced Industrial Science and Technology (AIST), Ibaraki, Japan \\ ${ }^{3}$ Institute of Solid State Physics, University of Tokyo, Chiba, Japan \\ Email: ${ }^{*}$ kanazawa@ u-gakugei.ac.jp
}

Received 12 November 2014

\begin{abstract}
The positron annihilation lifetimes and the Doppler broadening by slow positron beam are measured in thin Fe films with thickness $500 \mathrm{~nm}$, a thin $\mathrm{Hf}$ film with thickness $100 \mathrm{~nm}$, and the bilayer Fe $(50 \mathrm{~nm}) / \mathrm{Hf}(50 \mathrm{~nm})$ on quartz glass substrate. We have analyzed the behavior in vacancy-type defects in each layer through some deposition temperatures and annealing. It is observed that the thin Fe film, the thin $\mathrm{Hf}$ film, and the bilayer Fe $(50 \mathrm{~nm}) / \mathrm{Hf}(50 \mathrm{~nm})$ already contain many vacancy-type defects. We have investigated the change of densities of the vacancy-carbon complex and the small vacancy-cluster with carbons, through solid-state amorphization of Fe $(50 \mathrm{~nm}) / \mathrm{Hf}(50$ nm) bilayer.
\end{abstract}

\section{Keywords}

Metallic Films, Positron Annihilation Measurement, Solid-State Reaction, Fe Film, Diffusion, Vacancy-Type Defects

\section{Introduction}

Schwarz and Johnson [1] first have reported that isothermal annealing of thin film multilayers of two different crystalline metals ( $\mathrm{La}$ and $\mathrm{Au}$ ) can lead to the formation of an amorphous alloy. There exists a series of systems, mostly on the basis of one of the early transition metals and one of the late transition metals, in which the amorphous systems based on $\mathrm{Hf}$ and $\mathrm{Zr}$ have the advantage of a higher glass transition temperature. In addition, amorphization by mechanical alloying, a kind of solid-state amorphization, has been discovered [2].

It has been proposed that a multilayer structure, which is created in the initial stage of mechanical allying, plays an important role in amorphization [3]. The anomalously fast diffusion of one component and a large negative heat of mixing in the alloy are important factors in solid-state amorphization [4].

"Corresponding author.

How to cite this paper: Yamada, K., Sasaki, T., Nagata, T., Kanazawa, I., Suzuki, R., Ohdaira, T., Nozawa, K. and Komori, F. (2015) Solid-State Reaction and Vacancy-Type Defects in Bilayer Fe/Hf Studied by the Slow Positron Beam. Journal of Applied Mathematics and Physics, 3, 233-239. http://dx.doi.org/10.4236/jamp.2015.32034 
Oguchi et al. [5] have studied the solid-state amorphization in $\mathrm{Cu} / \mathrm{Hf}$ mutilayers by time different perturbed angular correlation (TDPAC). TDPAC and slow positron beam technique have shown that a strong distortion exists and many vacancy-type defects are present in the Hf layers of $\mathrm{Cu} / \mathrm{Hf}$ multilayers [6] [7]. Oshima et al. [8] have studied the solid-state amorphization in $\mathrm{Ni} / \mathrm{Hf}$ multilayers by slow positron beam technique. It has been shown that a high density of vacancy-type defects in Hf layers strongly induces a fast diffusion of Ni atoms, which promotes the solid-state amorphization. Fe/Hf multilayers, which have a soft magnetic property, have intensively been studied for high saturation magnetization and high density recording head materials [9]-[14]. A reason for the good magnetic softness is that these multilayers are in the nanocrystalline structures resulting in decreasing magnetocrystalline structure, suppressing the growth of columnar Fe grains have been required. In addition, thermal stability of these multilayers is very important, because most of magnetic heads are fabricated by glass bonding at high temperature. The growth of Fe grains and formation of amorphous-like interfacial product phase with the solid-state reaction between Fe and Hf layers are strongly related to the presence of vacancy-type defects in each layer. The variable energy slow positron beams have enabled studies of atomic vacancy-type defects at the surface, in the near surface, and in thin films [8] [15]-[19]. Especially the lifetime method with slow positron beam can supply the important information, that is, what kind of vacancy-type defects is contained in the thin films [20] [21]. In this study, we have performed the lifetime and the Doppler broadening measurements with the variable energy slow positron beams for the thin Fe film (500 nm), the thin Hf film (100 $\mathrm{nm})$, and the thin bilayer Fe $(50 \mathrm{~nm}) / \mathrm{Hf}(50 \mathrm{~nm})$, and have investigated the nature of the vacancy-type defects in these thin films.

\section{Experimental Method and Analysis}

The specimens were deposited by an MBE method using two electron gun sources for Fe and Hf. The MBE chamber was brought into the 10-10 Torr range prior to deposition. Thin Fe films of thickness $500 \mathrm{~nm}$ were deposited on the quartz glass substrate at room temperature and $473 \mathrm{~K}$. A thin Hf film of thickness $100 \mathrm{~nm}$ was deposited on the quartz glass substrate at room temperature. In the case of Fe/Hf bilayers the Hf layer of thickness $50 \mathrm{~nm}$ was deposited on a quartz glass substrate at room temperature and subsequently the Fe layer of thickness $50 \mathrm{~nm}$ was deposited on the Hf layer at room temperature. The incident positron energy E was variable from 0 to $13 \mathrm{keV}$. The measurements of Doppler broadening spectra by slow positron beam were carried out at room temperature with a high purity germanium detector. In order to estimate the vacancy-type defects in these thin films the S-parameter was used. S was determined by the ratio of the central area over 10 channels to the total area of the positron annihilation photopeak spectrum after subtracting the background.

The positron annihilation lifetime measurements by slow positron beam were carried out at room temperature by use of the positron pulsing system with an intense slow positron beam generated by an electron linac in the National Institute of Advanced Industrial Science and Technology (AIST) LINAC facility. The lifetime spectra were obtained by measuring the time interval between the timing signal derived electrically from the pulsing system and the timing signal of an annihilation $\gamma$-ray detected with a BaF2 scintillation detector. The detailed lifetime measurement system is described elsewhere [22]. For each spectrum, at least $3.0 \times 105$ counts were analyzed using the "RESOLUTION" routine [23] with good variance of the fits with a time resolution of about 290 ps FWHM.

In the present study, we used the scaling method [24] [25] for analyzing the positron implantation profiles in these thin films. Taking into account the diffraction effect of positron around the interfaces in multilayers, the scaling function of the positron implantation profile is represented as follows:

$$
\begin{aligned}
\tilde{P}(E, z) & =\frac{\langle z(E)\rangle_{i} N_{p}(E) P(E, z)}{\left[1+A_{i, i-1}(E, z)+A_{i, i+1}(E, z)\right]} \\
= & N_{l m}\left(\frac{u}{C_{l m}}\right)^{l} \exp \left[\left(\frac{u}{C_{l m}}\right)^{m}\right]
\end{aligned}
$$

where

$$
u=\frac{\left(z-d_{i-1}\right)}{\langle z(E)\rangle}+\sum_{j=1}^{i=1} \frac{d_{j}-d_{j-1}}{\langle z(E)\rangle_{j}}
$$


and $d_{i}$ is the length from the surface to the $i$ th-layer. $N_{i m}$ is a normalized constant, $C_{i m}, l$ and $m$ are parameters which depend on the materials. $\langle z(E)\rangle$ is a mean implantation depth. The atomic number dependence of these parameters has already been obtained [24] [25].

The parameter $N_{p}(E)$ and $A$ are found to depend on the atomic number of the constituent elements, from Monte Carlo simulations [25]

$$
N_{p}(E) \equiv \frac{\left[1+A_{i, i-1}(E, z)+A_{i, i+1}(E, z)\right]}{\int_{0}^{\infty} \mathrm{d} z \tilde{P}(E, z)}
$$

$A_{i, i-1}(E, z)$ and $A_{i, i+1}(E, z)$ are represented as follows:

$$
\begin{gathered}
A_{i, i-1}(E, z)=\left(\eta_{i-1}^{+}-\eta_{i}^{+}\right) \exp \left[-\frac{\left(z-d_{i-1}\right)}{\langle z(E)\rangle_{i}}\right] \\
A_{i, i+1}(E, z)=\left(\eta_{i+1}^{+}-\eta_{i}^{+}\right) \exp \left[-\frac{\left(d_{i-1}-z\right)}{\langle z(E)\rangle_{i}}\right] \\
\eta_{i}^{+}=b_{1}-b_{2} \exp \left[-b_{3} E\right]
\end{gathered}
$$

where $b_{1}, b_{2}$ and $b_{3}$ are parameters, which are represented as function of the atomic number of materials.

\section{Results and Discussions}

Figure 1(a) shows the measured $S$-parameters versus the incident positron energy for the bilayer Fe $(50 \mathrm{~nm}) / \mathrm{Hf}$ $(50 \mathrm{~nm})$ on quartz glass substrate with the bilayer thickness $\lambda \sim 100 \mathrm{~nm}$. The closed circles show the values of $S$-parameter for the bilayer, which is deposited on quartz glass substrate at room temperature and subsequently the Fe layer $(50 \mathrm{~nm})$ is deposited on Hf layer at room temperature. The open circles show the values of $S$-parameter for the bilayer annealed for 2 hours at $800 \mathrm{~K}$. The double circles show the values of $S$-parameter for the bilayer annealed for 4 hours at $800 \mathrm{~K}$.

Figure 1(b) shows the implantation profiles in the bilayer Fe $(50 \mathrm{~nm}) / \mathrm{Hf}(50 \mathrm{~nm})$ on the quartz glass substrate for some incident positron energies. It is found that the values of $S$-parameter in the bilayer Fe $(50 \mathrm{~nm}) / \mathrm{Hf}(50$ $\mathrm{nm}$ ) annealed for 2 hours at $800 \mathrm{~K}$ decrease remarkably in the region of the incident energy from 2 to $3 \mathrm{keV}$, in comparison with those of as-deposited bilayer.

From the positron implantation profiles in Figure 1(b), it is seen that the S-parameter in the region from 2 to 3 $\mathrm{keV}$ reflects the property of the Fe layer dominantly. This means that vacancy-type defects in the Fe layer annihilate dominantly and vacancy-type defects in the Hf layer do not change much during annealing for 2 hours at $800 \mathrm{~K}$.

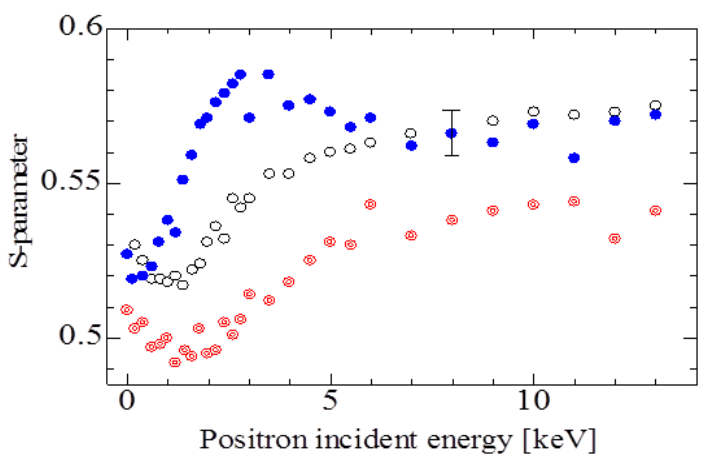

(a)

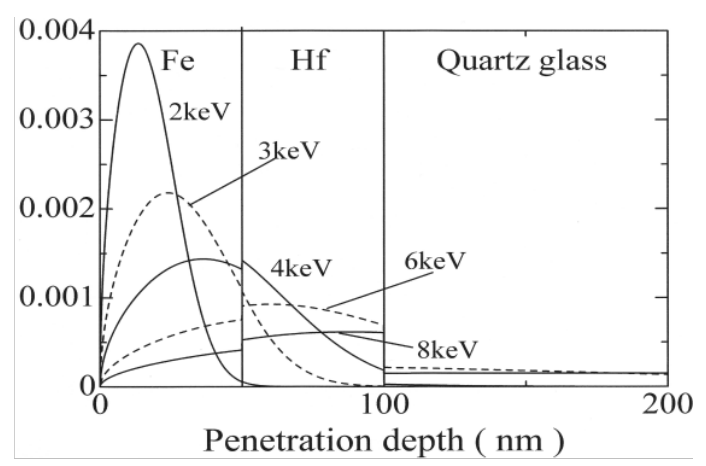

(b)

Figure 1. (a) S-parameter vs. incident positron energy for the bilayer Fe (50 nm)/Hf (50 nm); (b) The positron implantation profiles in the bilayer Fe $(50 \mathrm{~nm}) / \mathrm{Hf}(50 \mathrm{~nm})$ at some incident energies. 
Furthermore, it is found that the values of $S$-parameter in the bilayer annealed for 4 hours at $800 \mathrm{~K}$ decrease much in the region of the incident energy from $\sim 0$ to $\sim 13 \mathrm{keV}$, in comparison with those of as-deposited bilayer. This indicates that vacancy-type defects in both Fe and Hf layers annihilate much during annealing for 4 hours at $800 \mathrm{~K}$. These results show that diffusion of Fe component is much faster than that of Hf component. This corresponds to one of important factors in solid-state amorphization [4].

Figure 2 shows the positron annihilation lifetimes and those intensities in the thin Fe film of the thickness $500 \mathrm{~nm}$, which is grown on the quartz glass substrate at room temperature and $473 \mathrm{~K}$, and the lifetimes and intensities in the thin Hf film of the thickness $100 \mathrm{~nm}$, which is grown on the quartz glass substrate at room temperature. The positron incident energies are 6 and $3 \mathrm{keV}$ in the case of the Fe films and the Hf film, respectively. The lifetime, $\sim 332$ psec., in the thin Fe film of thickness $500 \mathrm{~nm}$, which is grown at room temperature, corresponds to vacancy-clusters, which contain carbon, compose of vacancies from $\sim 5$ to $\sim 10$ numbers [26]-[30]. On the other hand, the lifetime, $\sim 166$ psec., in the thin Fe film of thickness $500 \mathrm{~nm}$, which is grown at $473 \mathrm{~K}$, corresponds to the single-vacancies [26] [27], which might be trapped on carbon impurity atoms [29] [30], because single-vacancies move frequently in pure Fe at room temperature [31] [32]. The lifetime, 232 psec., in the thin Hf film of thickness $100 \mathrm{~nm}$ corresponds approximately to the single-vacancy [26].

Figure 3 shows the positron annihilation lifetimes and those intensities versus the incident positron energy for the bilayer Fe $(50 \mathrm{~nm}) / \mathrm{Hf}(50 \mathrm{~nm})$ after annealing for 4 hours at $800 \mathrm{~K}$. The lifetimes 277 psec. (the incident positron energy $2 \mathrm{keV}$ ) and 250 psec. (the incident positron energy $3 \mathrm{keV}$ ) correspond to vacancy-clusters composed of vacancies from 4 to 5 numbers. Now we shall consider interfacial reactions and growth of the interfacial product amorphous-phase. The free enthalpy, whose $T$ is temperature, is given for the amorphous $\mathrm{Hf}_{x} \mathrm{Fe}_{1-x}$ interfacial produce phase, assuming additivity of the specific heat as follows,

\section{Lifetime by slow positron beam}

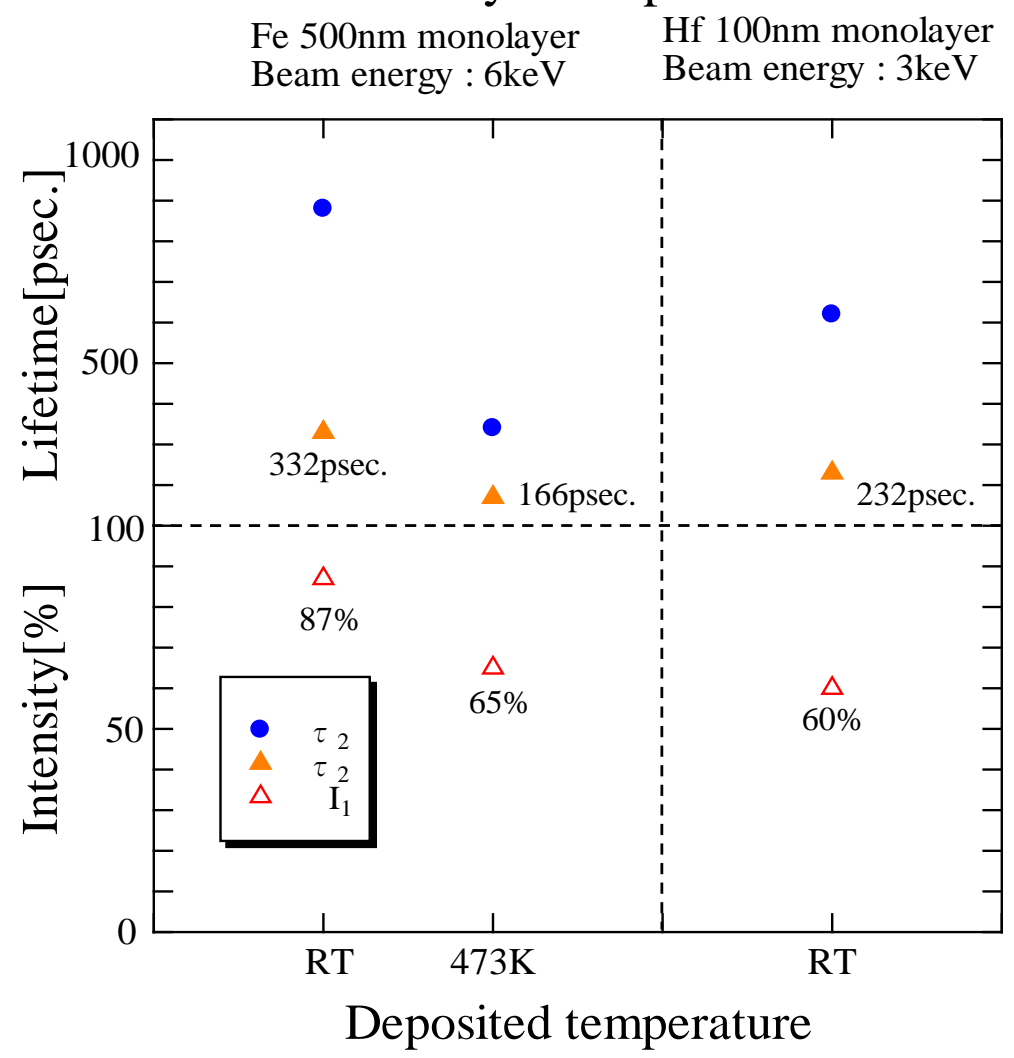

Figure 2. The positron annihilation lifetimes and those intensities in the thin Fe film of the thickness $500 \mathrm{~nm}$ at room temperature and $473 \mathrm{~K}$, and the lifetimes and intensities in the thin Hf film of the thickness $100 \mathrm{~nm}$ at room temperature. 


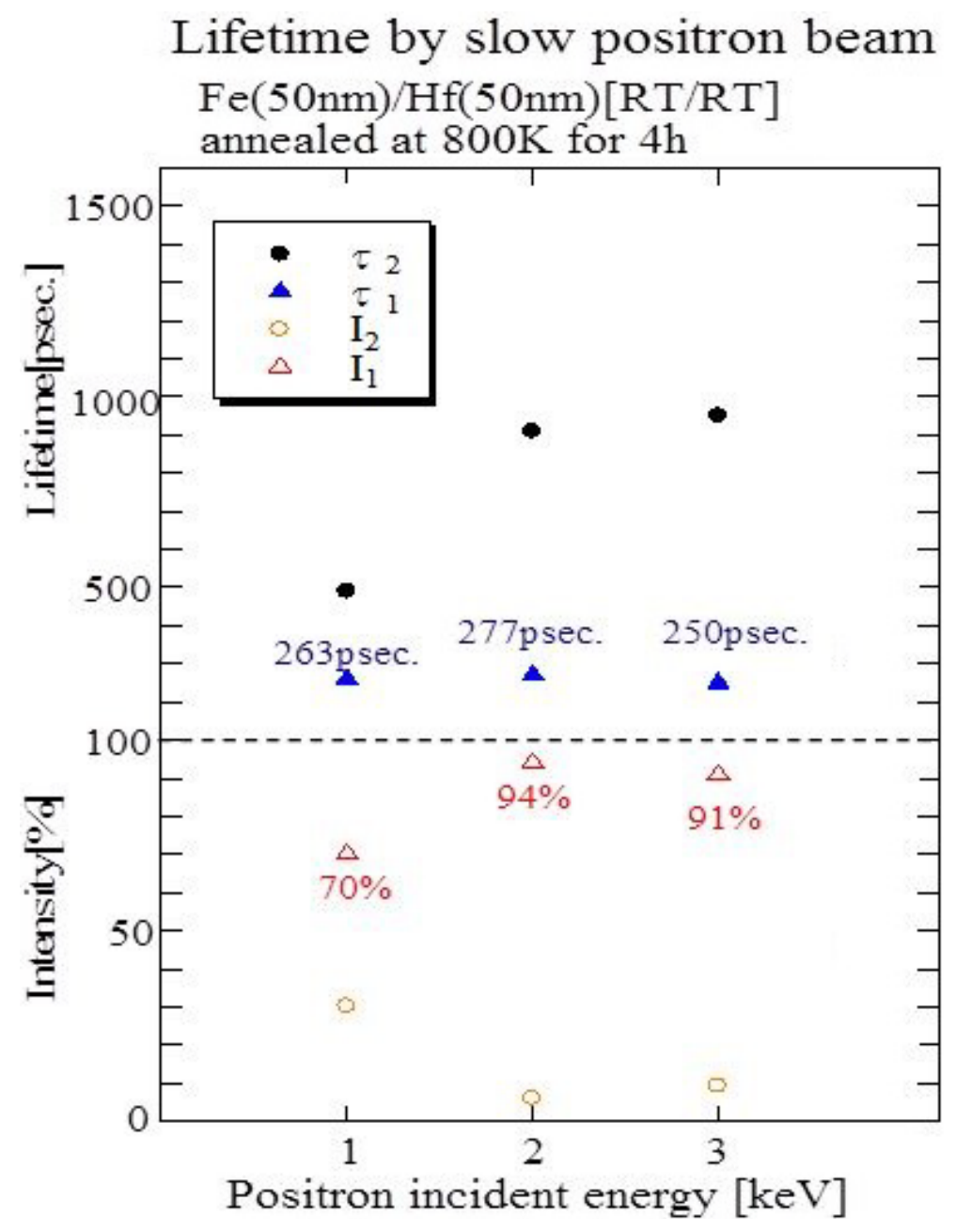

Figure 3. The positron annihilation lifetimes and those intensities vs. the incident positron energy for the bilayer Fe $(50 \mathrm{~nm}) / \mathrm{Hf}(50 \mathrm{~nm})$ after annealing for 4 hours at $800 \mathrm{~K}$.

$$
G(x, T)=x G^{\mathrm{Hf}}(T)+(1-x) G^{\mathrm{Fe}}(T)+\Delta H_{\text {mix }}-T \Delta S_{\text {mix }}
$$

$G^{\mathrm{Hf}}(T)$ and $G^{\mathrm{Fe}}(T)$ are the free enthalpies of Hf layers and Fe layers, respectively.

$\Delta H_{\text {mix }}$ is the enthalpy of mixing.

$\Delta S_{\text {mix }}$ is the entropy of mixing.

The free enthalpy of the amorphous $\mathrm{Hf}_{x} \mathrm{Fe}_{1-x}$ interfacial product phase is lowered due to the large negative heat of mixing. These terms give a driving force for the interfacial reaction. We must consider why the stable crystalline HfFe alloy phase is not formed at the beginning of the reaction although there is enough mobility to grow the amorphous HfFe interfacial product phase in a diffusion reaction. Kinetic restrictions are necessary so that the transition will not go into the equilibrium state, but instead to the metastable amorphous state. One answer has its basis in the asymmetric mobilities of the two reactants (Fe and Hf). Certainly the present experimental results show that the diffusion of $\mathrm{Fe}$ component is much faster than that of $\mathrm{Hf}$ component. Thus the smaller late transition metals (Fe, Co etc.) are responsible for the thermally excited mass transport in contrast to the early transition metals (Hf, Zr etc.), which move just out of their equilibrium positions but do not contribute to the diffusion process. Another problem is why the activation energy of the interfacial reaction is much low in comparison with the activation energy $(\sim 2.45 \mathrm{eV})$ [33] for the self-diffusion of Fe in bcc $\alpha$-Fe. The present experimental results give one answer for this problem. That is, $\mathrm{Fe} / \mathrm{Hf}$ layers already contain many vacancy-type 
defects. In the case of Fe layers, taking account of the carbon-vacancy binding energy ( $\sim 0.85 \mathrm{eV}$ ) [30], the activation energy is estimated to be $\sim 1.40 \mathrm{eV}=\sim 0.85 \mathrm{eV}+\sim 0.55 \mathrm{eV}$ (the vacancy migration energy in Fe) [30]. This value of the activation energy is much lower than the activation energy $(\sim 2.45 \mathrm{eV})$ of the self-diffusion of Fe.

\section{Conclusion}

We have measured the positron annihilation Dopper broadening in the bilayer Fe $(50 \mathrm{~nm}) / \mathrm{Hf}(50 \mathrm{~nm})$ by slow positron beam during the isochronal annealing at $800 \mathrm{~K}$. We have analyzed the changes in density of the vacancy-carbon complex and the small vacancy-cluster, which might contain carbons, through solid-state amorphization of Fe $(50 \mathrm{~nm}) / \mathrm{Hf}(50 \mathrm{~nm})$ with the positron annihilation lifetime by slow positron beam. We have discussed what kind of vacancy-type defects exists in Fe (500 nm) layer and Hf (100 nm), respectively.

\section{References}

[1] Schwarz, R.B. and Johnson, W.L. (1983) A Comparative Study of MR Imaging Profile of Titanium Pedicle Screws. Phys. Rev. Lett., 51, 415. http://dx.doi.org/10.1103/PhysRevLett.51.415

[2] Koch, C.C., Calxin, O.B., McCamey, C.G. and Scarbrough, J.O. (1983) Preparation of “Amorphous” $\mathrm{Ni}_{60} \mathrm{Nb}_{40}$ by Mechanical Alloying. Appl. Phys. Lett., 43, 1017. http://dx.doi.org/10.1063/1.94213

[3] Weeber, A.W. and Bakker, H. (1988) Amorphization by Ball Milling. A Review. Physica B, 153, 93. http://dx.doi.org/10.1016/0921-4526(88)90038-5

[4] Samwer, K. (1988) Amorphisation in Solid Metallic Systems. Phys. Rep., 161, 1. http://dx.doi.org/10.1016/0370-1573(88)90006-3

[5] Oguchi, T., Kanazawa, I., Iwashita, T., Soe, W., Yamaguchi, A. and Yamamoto, R. (1992) Solid State Amorphization in Cu-Hf Multilayers Studied by Time-Differential Perturbed-Angular Correlation. J. Non-Cryst. Solids., 150, 448. http://dx.doi.org/10.1016/0022-3093(92)90170-O

[6] Yamamoto, Y., Matsubara, H., Yamaguchi, M., Oguchi, T., Kanazawa, I., Iwashita, T., Ito, Y., Takamura, S., Soe, W., Yamaguchi, A. and Yamamoto, R. (1993) Cu-Hf Multilayers Studied by Slow Positron Beam and Time Differential Perturbed Angular Correlation. J. Magn. Magn. Mater., 126, 170. http://dx.doi.org/10.1016/0304-8853(93)90573-K

[7] Matsubara, H., Yamamoto, Y., Kanazawa, I., Iwashita, T., Ito, Y., Takamura, S., Soe, W. and Yamamoto, R. (1994) Studies of Multilayers of Cu-Hf with a Variable-Energy Positron Beam. Hyperfine Interaction, 84, 159. http://dx.doi.org/10.1007/BF02060657

[8] Oshima, N., Nakajyo, T., Kanazawa, I., Iwashita, T., Ito, Y., Soe, W. and Yamamoto, R. (1995) Multilayered Ni/Hf and Solid-State Amorphization Studied Using the Slow Positron Beam Technique. Appl. Surf. Sci., 85, 329. http://dx.doi.org/10.1016/0169-4332(94)00354-8

[9] Watanabe, Y., Oura, H. and Onozato, N. (1990) Magnetic Properties of Fe-Ta-N-O Film with High Saturation Flux Density. IEEE Trans. Magn., 26, 1500. http://dx.doi.org/10.1109/20.104429

[10] Hasegawa, N. and Saito, M. (1990) Soft Magnetic Properties of Microcrystalline Fe-M-C (M=V, Nb, Ta) Films with High Thermal Stability. J. Magn. Soc. Jpn., 14, 313. http://dx.doi.org/10.3379/jmsjmag.14.313

[11] Taneko, N., Shimada, Y., Fukamichi, K. and Miyakawa, C. (1991) Fabrication of Fe-Zr-N Films with Very Low Coercivity. Jpn. J. Appl. Phys., 30, L195. http://dx.doi.org/10.1143/JJAP.30.L195

[12] Hasegawa, N., Kataoka, N., Fujimori, H. and Saito, M. (1991) Structural and Soft Magnetic Properties of Fe/Fe-Hf-C Multilayered Films with the High B s and Thermal Stability. J. Appl. Phys., 70, 6253. http://dx.doi.org/10.1063/1.349983

[13] Hoffmann, H. (1964) Quantitative Calculation of the Magnetic Ripple of Uniaxial Thin Permalloy Films. J. Appl. Phys., 35, 1790. http://dx.doi.org/10.1063/1.1713743

[14] Herzer, G. (1990) Grain Size Dependence of Coercivity and Permeability in Nanocrystalline Ferromagnets. IEEE Trans. Magn., 26, 1397. http://dx.doi.org/10.1109/20.104389

[15] Schultz, P.J. and Lynn, K.G. (1988) Interaction of Positron Beams with Surfaces, Thin Films, and Interfaces. Rev. Mod. Phys, 60, 701. http://dx.doi.org/10.1103/RevModPhys.60.701

[16] Murashige, Y., Tashiro, M., Nakajyo, T., Koizumi, T., Kanazawa, I., Komori, F. and Ito, Y. (1997) The Multilayer Fe/Hf Studied with Slow Positron Beam. Appl. Surf. Sci., 113-114, 169. http://dx.doi.org/10.1016/S0169-4332(96)00844-6

[17] Tashiro, M., Nakajyo, T., Murashige, Y., Koizumi, T., Kanazawa, I., Komori, F., Soe, W., Yamamoto, R. and Ito, Y. (1997) The Study of Multilayers Fe/Hf and Ni/Hf by Slow Positron Beam Technique. Appl. Surf. Sci., 116, 263. 
http://dx.doi.org/10.1016/S0169-4332(96)01066-5

[18] Ohtaki, H., Koizumi, T., Iwamoto, A., Kanazawa, I., Komori, F. and Ito, Y. (1998) The Study of Amorphization in Multilayer Fe/Hf with Slow Positron Beam. Appl. Surf. Sci., 130-132, 828. http://dx.doi.org/10.1016/S0169-4332(98)00161-5

[19] Nagata, T., Ozaki, A., Terashima, Y., Kanazawa, I., Suzuki, R., Ohdaira, T., Komori, F. and Ito, Y. (2000) Solid-State Amorphization in Multilayer Fe/Hf Studied by Slow Positrons. Nucl. Instrument Method. Phys. Research B, 170, 240. http://dx.doi.org/10.1016/S0168-583X(00)00162-2

[20] Takagi, K., Furukawa, N., Kanazawa, I., Suzuki, R. and Ohdaira, T. (2002) Formation of Hydrogen-Induced Vacancies during Growth of the Fe Layer Studied by Slow Positron Beam. Surf. Sci., 514, 298. http://dx.doi.org/10.1016/S0039-6028(02)01644-8

[21] Suzuki, H., Azami, K., Komagata, S., Kanazawa, I., Suzuki, R. and Ohdaira, T. (2010) Diffusion of Positrons in Polymers Studied by Slow Positron Beam. J. Phys. CS, 225, 012053.

[22] Suzuki, R., Kobayashi, Y., Mikado, T., Ohgaki, H., Chiwaki, M., Yamazaki, T. and Tomimasu, T. (1991) Slow Positron Pulsing System for Variable Energy Positron Lifetime Spectroscopy. Jpn. J. Appl. Phys., 30, L532. http://dx.doi.org/10.1143/JJAP.30.L532

[23] Kirkegaard, P., Eldrup, M., Mogensen, O.E. and Pedersen, N.J. (1981) Program System for Analysing Positron Lifetime Spectra and Angular Correlation Curves. Comput. Phys. Commun., 23, 307. http://dx.doi.org/10.1016/0010-4655(81)90006-0

[24] Ghosh, V.J. (1995) Positron Implantation Profiles in Elemental and Multilayer Systems. Appl. Surf. Sci., 85, 187. http://dx.doi.org/10.1016/0169-4332(94)00331-9

[25] Aers, G.C., Marshall, P.A., Leung, T.C. and Goldberg, R.D. (1995) Defect Profiling in Multilayered Systems Using Mean Depth Scaling. Appl. Surf. Sci., 85, 196. http://dx.doi.org/10.1016/0169-4332(94)00332-7

[26] Puska, M.J. and Nieminen, R.M. (1994) Theory of Positrons in Solids and on Solid Surfaces. Rev. Mod. Phys., 66, 841. http://dx.doi.org/10.1103/RevModPhys.66.841

[27] Nieminen, M.R. and Puska, M.J. (1982) Defect Spectroscopy with Positrons: Calculations. Coleman, P.G., Sharma, S.C. and Dian, L.M., Eds., Proc. $6^{\text {th }}$ Int. Conf. Positron Annihilation, North-Holland Pub, 395.

[28] Kamimura, Y., Tsutsumi, T. and Kuramoto, E. (1995) Calculations of Positron Lifetimes in a Jog and Vacancies on an Edge-Dislocation Line in Fe. Phys. Rev. B, 52, 879. http://dx.doi.org/10.1103/PhysRevB.52.879

[29] Hautojärvi, P., Johansson, J., Vehanen, A., Yli-Kauppila, J. and Moser, P. (1980) Vacancy-Carbon Interaction in Iron. Phys. Rev. Lett., 44, 1326. http://dx.doi.org/10.1103/PhysRevLett.44.1326

[30] Vehanen, A., Hautojärvi, P., Johansson, J., Yli-Kauppila, J. and Moser, P. (1982) Vacancies and Carbon Impurities in $\alpha$-Iron: Electron Irradiation. Phys. Rev. B, 25, 762. http://dx.doi.org/10.1103/PhysRevB.25.762

[31] Tanigawa, S., Hinode, K., Owada, N., Doyama, M. and Okuda, S. (1979) The Studies of Recovery of Irradiated Iron by Positron Annihilation. Hasiguchi, R.R. and Fujiwara, K., Proc. 5th Int. Conf. Positron Annihilation, Japan Institute of Metals, Sendai, 501.

[32] Hautojärvi, P., Judin, T., Vehanen, A., Yli-Kauppila, J., Johansson, J., Verdone, J. and Moser, P. (1979) Annealing of Vacancies in Electron-Irradiated $\alpha$-Iron. Solid State Commun., 29, 855. http://dx.doi.org/10.1016/0038-1098(79)90507-6

[33] Hettich, G., Mehrer, H. and Maier, K. (1977) Self-Diffusion in Ferromagnetic $\alpha$-Iron. Scripta Met., $11,795$. http://dx.doi.org/10.1016/0036-9748(77)90078-3 